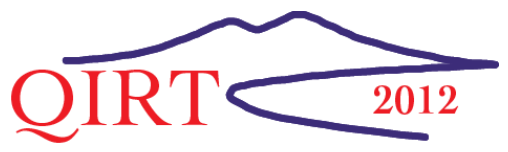

\title{
Production integrated NDT by means of automated Thermography
}

by T. Schmidt* ${ }^{\star}$ S. Dutta* and T. Ullmann**

*German Aerospace Center, Institute of Structures and Design, Center for Leightweight-Production-Technology, Alter Postweg 101, 86159 Augsburg, Mobile: +49-172-8343570 (Schmidt); +49-173-5617742 (Dutta); Fax:+49-821-5985883, t.schmidt@dlr.de, somen.dutta@dlr.de

**German Aerospace Center, Institute of Structures and Design, Pfaffenwaldring 38-40, 70569 Stuttgart, Phone: +49-711-68628497, Fax: +49-711-6862-227, thomas.ullmann@dlr.de

\section{Abstract}

Since the last decade the demand of CFRP parts is rapidly increasing in the aerospace industry. Besides the challenges in design and engineering, the manufacturing quality plays a key role. In order to achieve a higher quality, to decrease scrap rate and lowering manufacturing costs, the need for process integrated measurement techniques such as Lock-In Thermography are obligatory. One of the deficits so far is that Thermography lacks implementation on industrial scale. The approach at DLR Augsburg is a robot based measuring system for industrial applications such as measuring large CFRP structures of future aircraft generations (see Fig 1). Research at the DLR-Center for LightweightProduction-Technology in Augsburg focuses on this and will give insight into the challenges and current work with this paper.

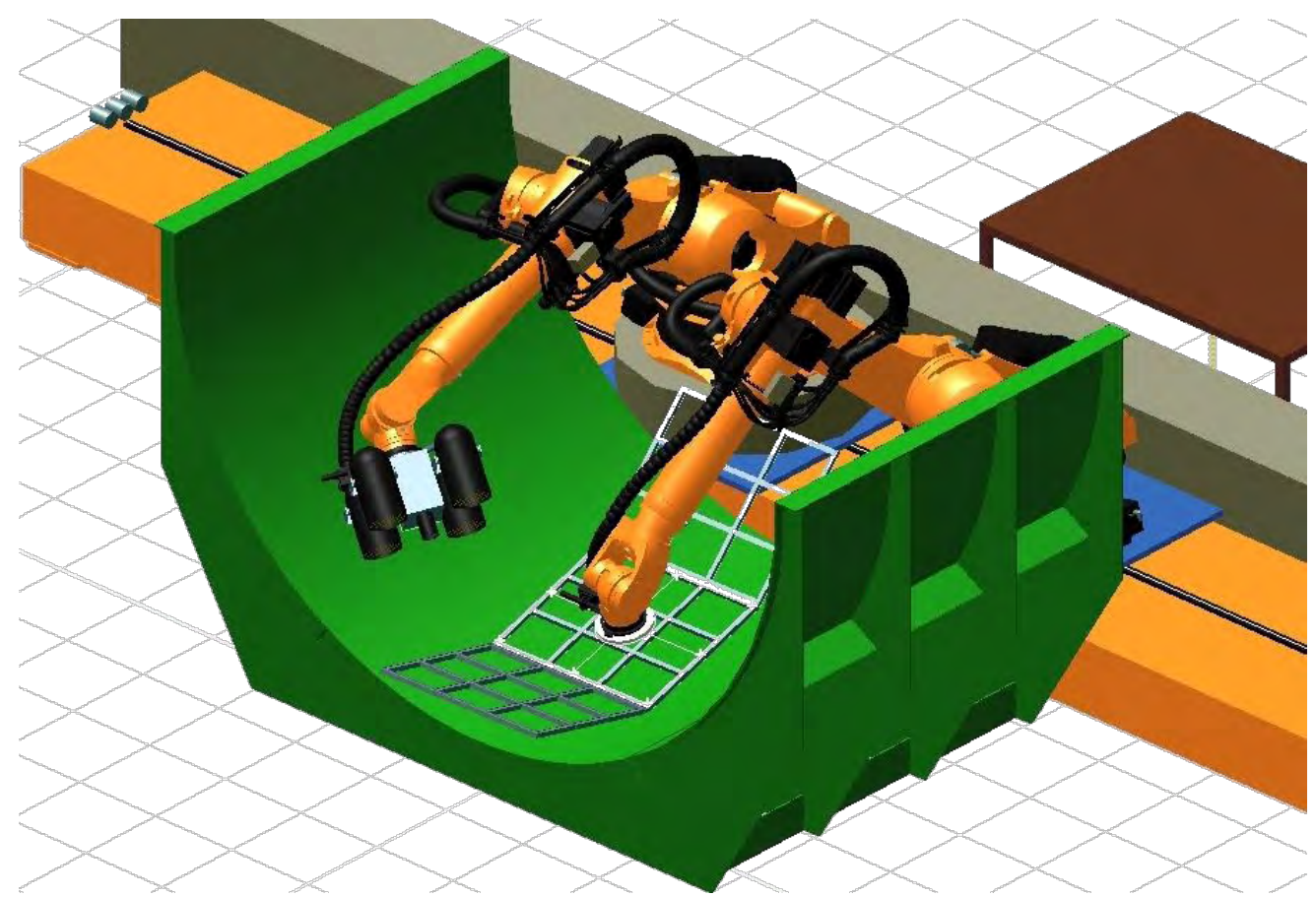

Fig 1 Scenario of automated thermography within production environment 


\section{Introduction}

Over the last decades there has been enormous growth in transportation, particularly in aerospace. Therefore in civil and military aircraft $(\mathrm{A} / \mathrm{C})$ there had been a major change in design and material choice. Fig 2 depicts the growth of CFRP usage on structural weight in percent over the last decades. Especially the last 10 years had been a big step up which puts out new challenges for the entire industry. Starting with the design and analysis of a new aircraft, engineers had to develop new strategies and tools to cover the requirements of modern composite materials. This in return puts manufacturing and quality assurance (QA) also into new position. Processes for a metallic airframe were obsolete and modern composite knowledge had to find its way into production. Even more dramatic, that these composite processes bring a lot more responsibilities to the manufacturer. Compared to metal technology it is more or less like a master forming that takes places in your workshop instead of your suppliers. There are a lot more process steps involved and number of parameters is long. Where just to say CFRP would be generalizing. There are two major technology areas which are named by the used matrix system which are thermoplastic and thermoset resins. Each of them has a few more branches. But just to name prepreg and the textile/infusion technology for the thermosets. In a nutshell all these processes involve many steps and don't have a similar robustness compared to a production using metals.

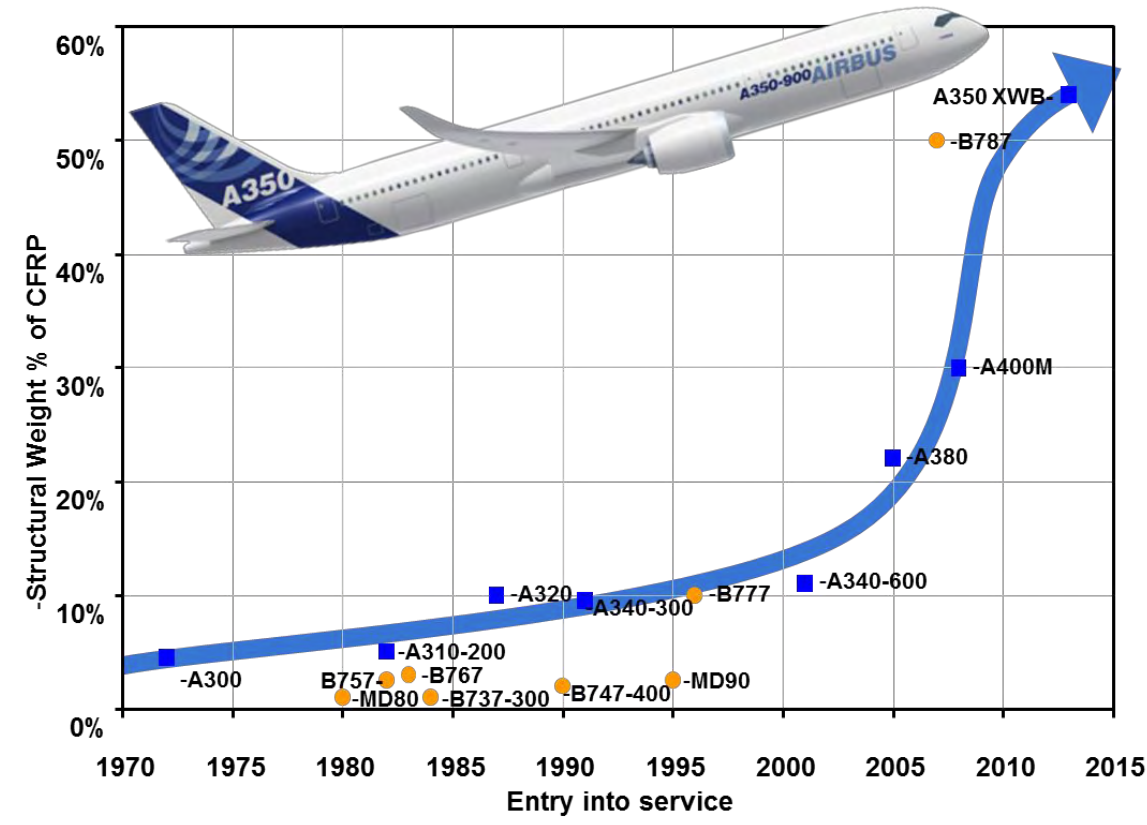

Fig 2 Increase in CFRP in commercial and military aircraft (Source: Airbus)

Focusing on only one type of aircraft, in our case the next generation Airbus A350XWB (see Fig 3), the numbers are overwhelming. Looking at a prospected number of aircraft per year with a classical fuselage design consisting of shells, stringers, frames and beams, the numbers are tremendous. The order books outline a yearly production rate of round about $480 \mathrm{~A} / \mathrm{C}$. Consequently the same numbers for wings, engine casings, vertical and horizontal tail planes are inherent. The pure number seems comparably low for other industries but in aerospace there is still a high amount of manual labor. On the other hand, although it is a global market with its two OEMs (Airbus and Boeing) for this type of aircraft, the list of suppliers that can cover this demand is not very long. One can observe that a change in composite production towards automation has already been started. But again, these are new technologies on a material that has a 40 year history. Besides that aerospace industry has a very strict regime on quality assurance which is obvious because safety is one of the most important measures. All this adds up to a development with a long lead time, costly processes due to high amount of manual labor and therefore a high scrap rate and finally a too small throughput. Exactly these issues are to address in the near future to make the use of composites successful.

Saying this for production also covers quality assurance. There is an enormous demand on non-destructive testing (NDT) of all these composite structures in the next few years. The NDT methods need to be highly accurate, flexible and defect selective. Additionally all NDT technologies need to be certified to withstand aerospace requirements as well as the ability to deliver the same throughput as production. Otherwise NDT ends up as a bottleneck that holds up the assembly of structures and then produces high costs. Using NDT methods in a wider range actually could support the development of robust production processes in the future. 


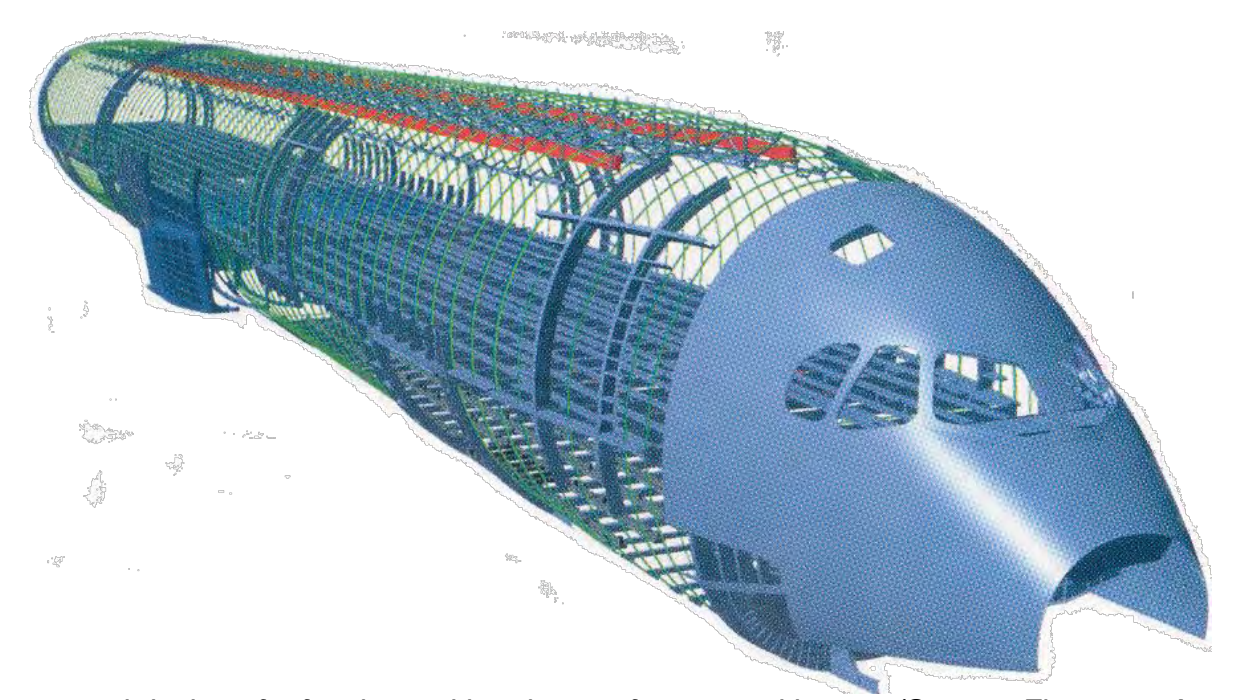

Fig 3 structural design of a fuselage with stringers, frames and beams (Source: Flugrevue A350XWB)

\section{State of the Art in Aerospace}

Fig 4 shows the classical production process for the textile/ infusion technology. Most of the process steps are still manual work as described above. Inspection in between each step is only visual or acoustic on a very qualitative scale. Quality assurance takes place but is more or less only documentation if each step had been done, but neither how accurate it was nor what size or location of anomaly had been detected.

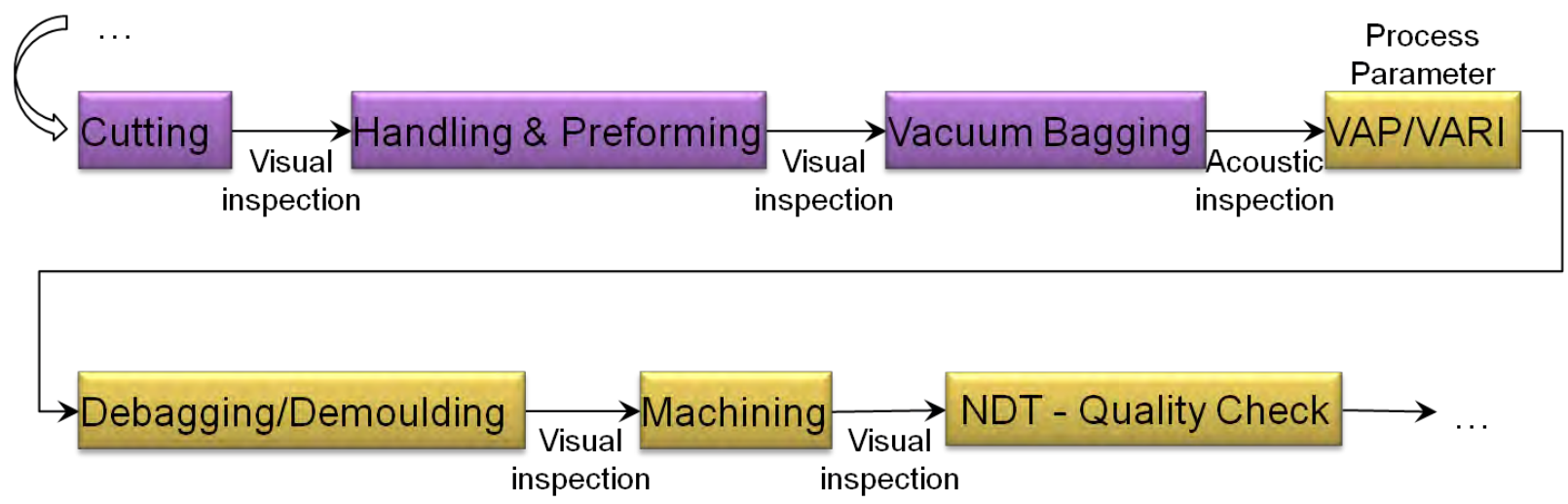

Fig 4 Present production strategy in textile/infusion technology

The final quality check is usually performed by classical ultrasonic inspection. In nearly all cases water coupled ultrasonic inspection is the choice. The inspection machines reach from gantry solutions with linear and rotational axis using submersion and squirter technology. Sampled phased array in transmission and pulse/echo finds a very wide spread. Examples for each technology as shown below. 

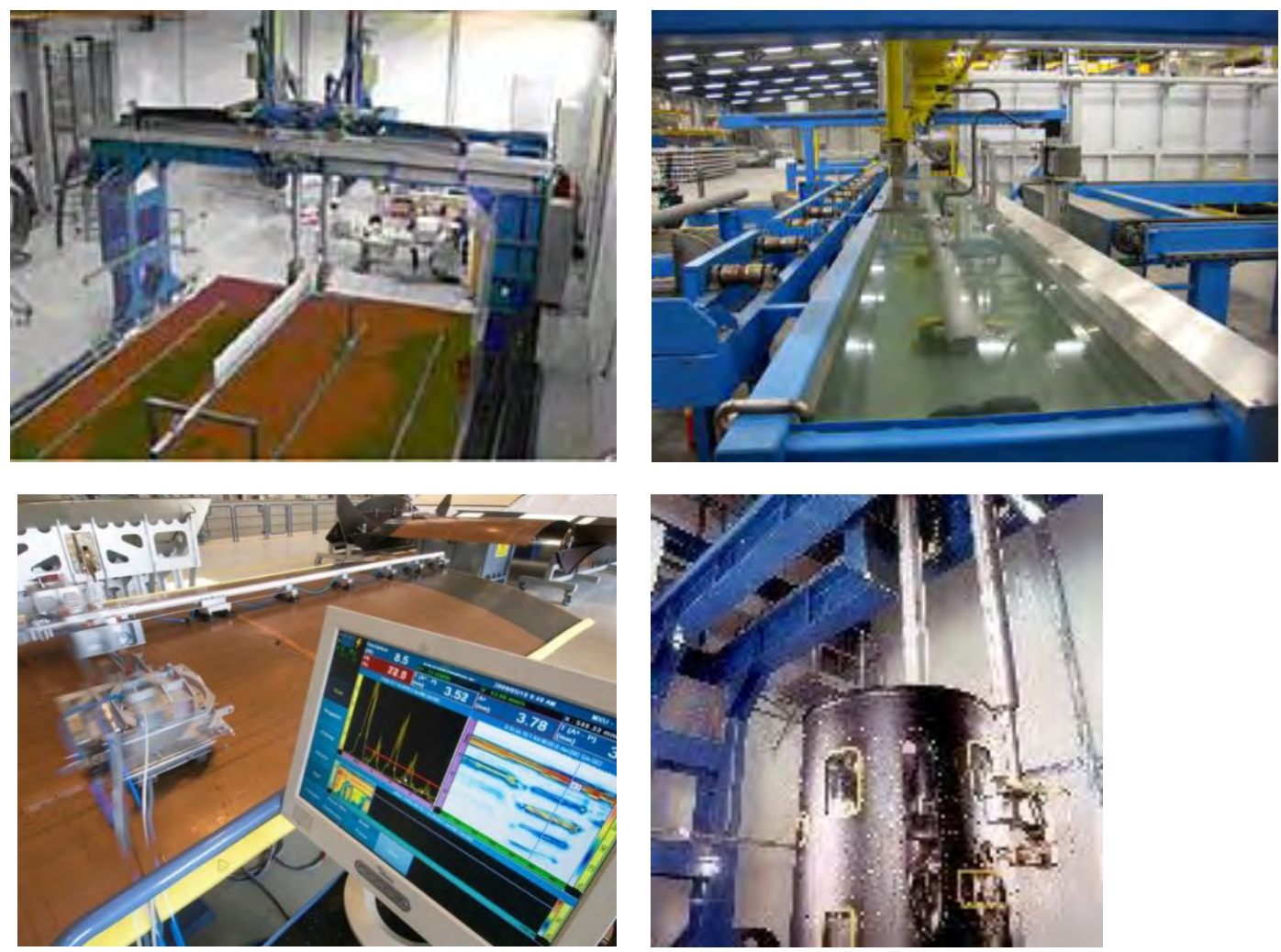

Fig 5 Examples for water coupled ultrasonic inspection (sources: National Composites Network, Premium Aerotec, IntelligeNDT, HPI)

\section{Challenges for NDT}

Besides final inspection of Components, NDT can be used during manufacturing in terms of monitoring and even control of process quality. Picking up the drawbacks from nowadays quality assurance for existing production scenarios one can identify the challenges for future NDT. NDT has to deliver proper indicators to evaluate the quality of a part or structure. Quality control with NDT combines the advantages of both classical methods of process control. On the one hand the collected statistics data will assist to develop the manufacturing process and on the other hand monitoring of process variables. The Aerospace industry relies on non-destructive testing (NDT) methods to detect and characterise the manufacturing failure and damages. As a part of the production integrated NDT for CFRP components it is necessary to automate the NDT Inspections system. Taking the knowledge that derives from a detailed documentation it allows to improve the manufacturing process know how. This should lead to a closed engineering loop and therefore a continuous improvement. Consequently an improved rework rate is the outcome.

Due to the lack of fully an automatic production chain, the industry is presently not being able to fulfill the drastically increasing demand of the components. All items mentioned above will put pressure on the development of future NDT to achieve the desired cost efficiency. The goal is to improve the automation level in the manufacturing technology, reduce the manufacturing cost and improve the part's quality. An improved rework rate will lead towards a higher output rate of production. It might actually be imaginable to decrease the amount of testing at the final stage. A final quality approval based on a few samples or random checks could be possible. Such a major change in a QA strategy needs to be certified for sure. Nevertheless a higher degree of flexible automation in measurement and evaluation is necessary to enable a continuous improvement. An integrated production and quality assurance rather than local improvements seem to play a key role.

Projecting this on the process chain mentioned earlier, research at ZLP Augsburg aims at two process steps that want to be covered with current work as depicted in Fig 6. 

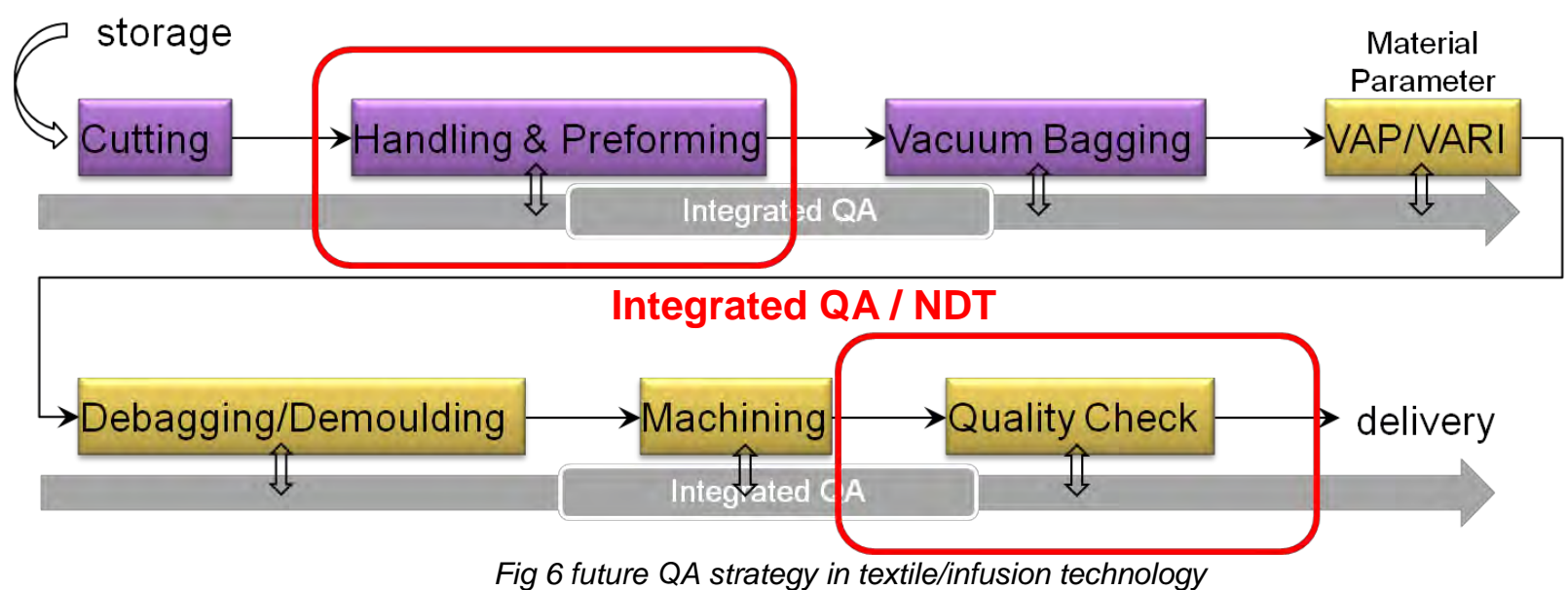

Every processing step in the production line should be monitored to improve the added value without derogating the quality of the components. Implementing new measuring and evaluation technology throughout the production line could be the key to avoid or minimize the manufacturing irregularity. One such Measurement system must meet the following criteria like being contactless, having higher measurement speed as well as easy-to-interpret, being automatic and obviously economic. Inspection tools also must meet measurement specifications for complex and large components at high speeds. On the other side process integrated NDT has to fulfil the requirements of industrial production concerning integratebility and data Management.

There are several approaches of non-destructive measuring method, which are applied here in DLR to expose the complete manufacturing process. This paper is focusing on automated non-destructive Thermography method, which exposes the "handling \& preforming" and as well as component "quality check".

\section{Approach - production integrated, automated Thermography as a choice}

The future challenges are the basics for the approach. Searching a NDT method that is suitable to cover all needs, such as contactless, accurate, of high performance, able to integrate into existing production scenarios, research at ZLP Augsburg had been focused on active Thermography. Using industrial robots as manipulators one could achieve a very flexible and highly accurate solution with the highest rate of automation as shown in Fig 7 .

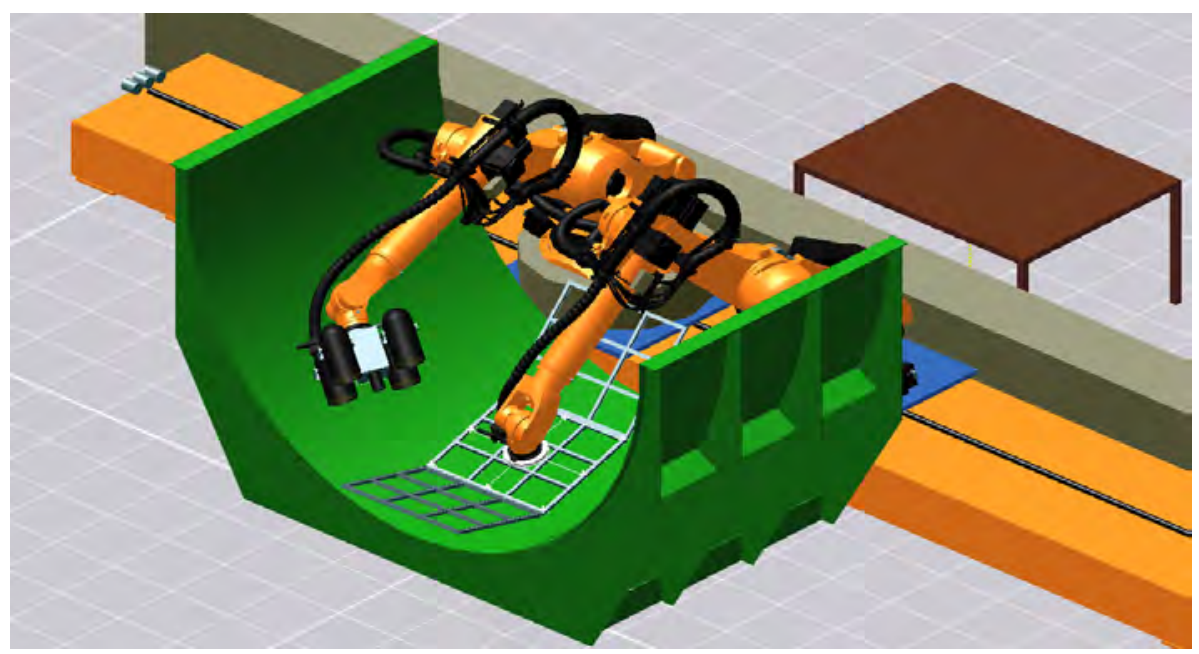

Fig 7 Approach - production integrated, automated Thermography

The choice on active Thermography has been made because it is capable to visualize defects on dry carbon fiber performs as well as cured parts. This capability fulfills the requirements to detect features like missing rovings, fuzzballs or even the fiber orientation on textile performs. The more common use is to detect anomalies in cured parts such as delaminations, dry spots, porosity or disbondings. Examples for both cases are shown in the following Fig 8. There are more applications imaginable like using Thermography to survey the process of infusion and how the flow front evolves. But is not a prime topic that will be investigated. 


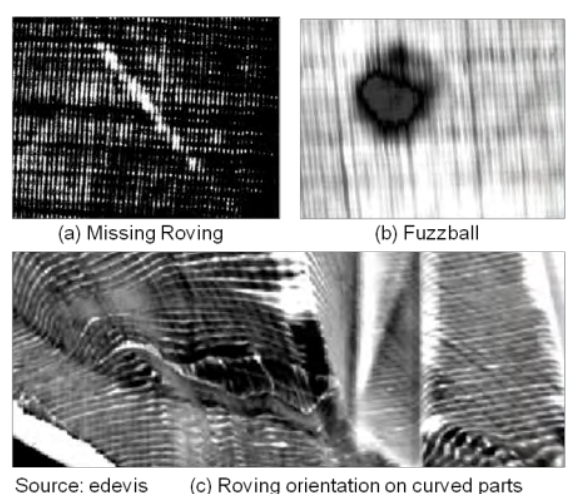

Fig 8 Examples for measuring carbon fibre performs $(a, b, c)$ and cured parts (d)

The choice on industrial robots is self evident as they are used as manipulators in our production environment as well. Through their kinematics they allow a high degree of flexibility combined with an adequate accuracy. Not to mention, that both features are the determining factor especially as a standard industrial robot is comparably inexpensive.

\section{Realization}

At ZLP Augsburg, a highly flexible robotic work cell for future composite production has been developed and built. This work cell allows research on an industrial scale as the dimensions for possible structures are enormous. The work cell consists of 4 major gantry portals. Each of them has move- and tiltable vertical axis with a robotic arm at its end. The entire working volume can be split in quadrants to allow parallel work on different projects. The main goal is to demonstrate and perform active Thermography within this work cell. For a detailed view, please refer to Fig 9.
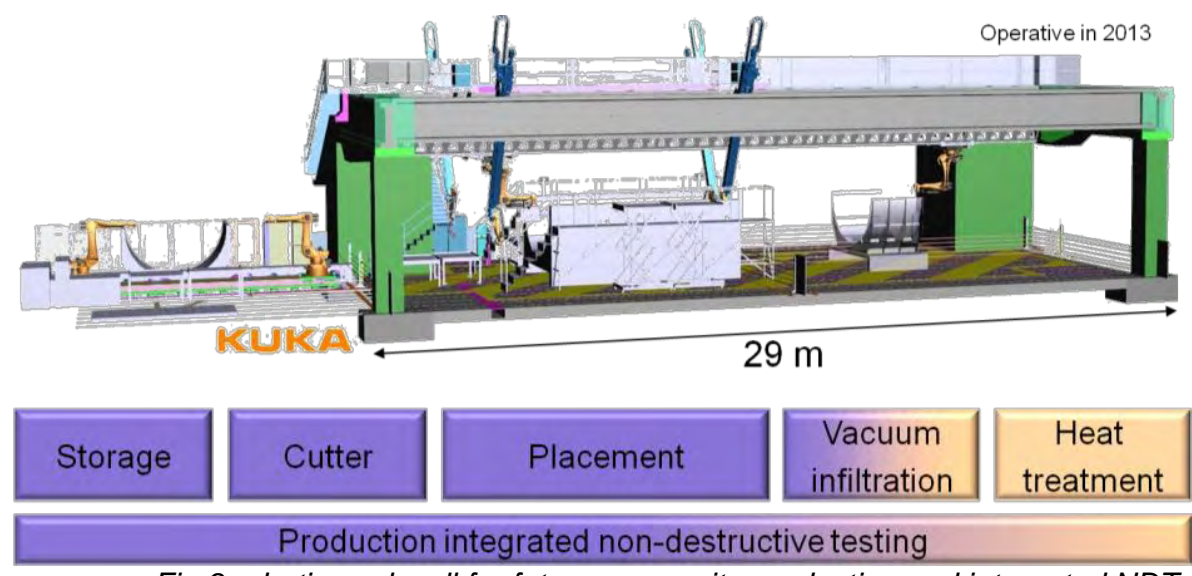

Fig 9 robotic work cell for future composite production and integrated NDT

As the above mentioned work cell is huge in its dimension, the decision has been made to proceed in several steps. One of the reasons is risk mitigation; therefore first concepts had been started on a smaller scale. As shown in

Fig 10 integration of the Thermography setup has been started on a work cell that consists of a linear axis which is mounted on the ground. The linear axis carries to robots. Both have a nominal reach of R3000 at a load capacity of $210 \mathrm{~kg}$. The developed Thermography measuring system based on industrial robots carries the halogen lamps and IR camera. This measuring system is designed to achieve homogenous excited measuring filed and convenient to measure for 3D complex components. 


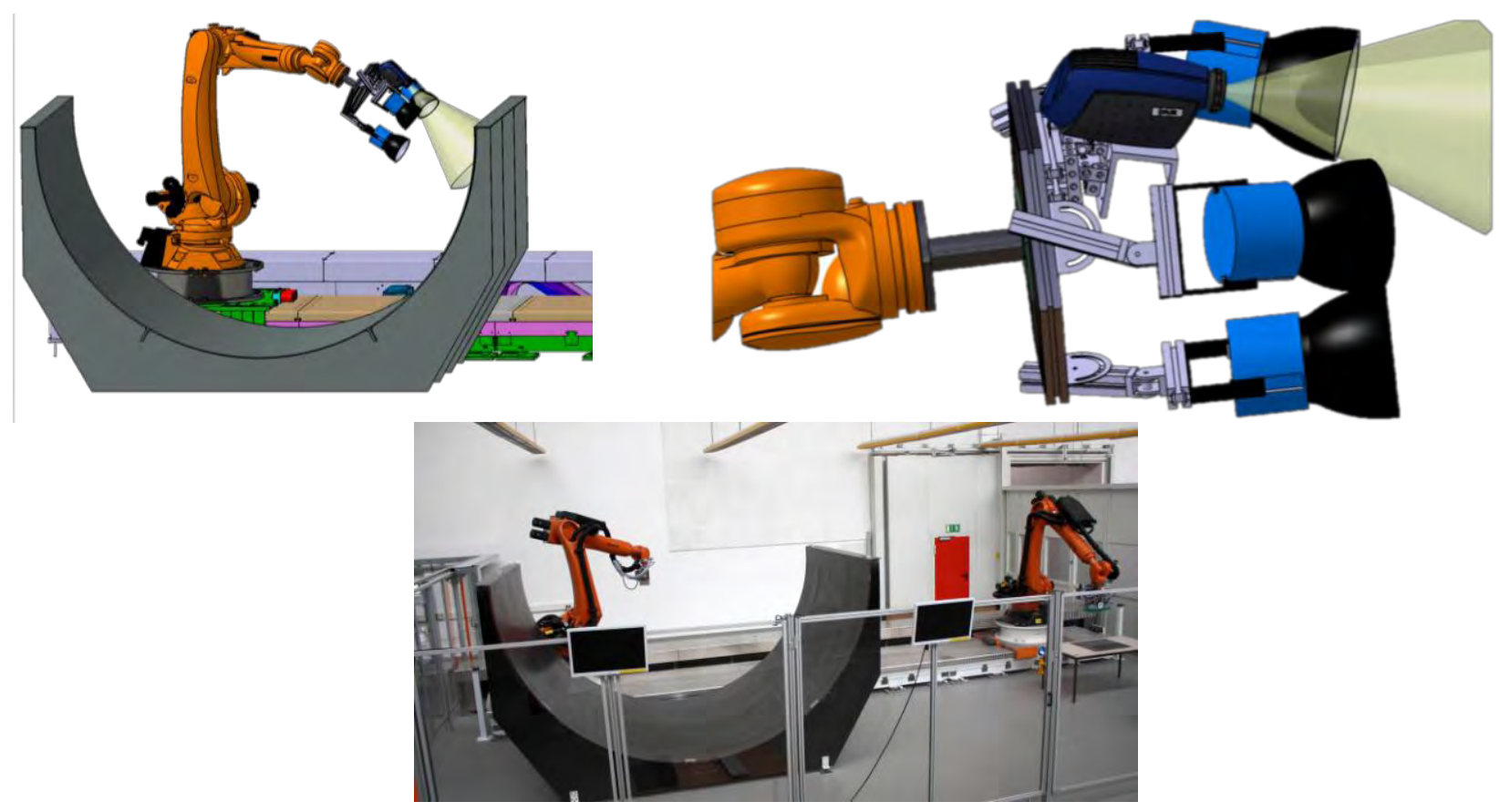

Fig 10 Experimental set up for risk mitigation

As a matter of principle there are a number of scenarios possible to integrate a system for active Thermography on the shown robot. All concepts can be categorized in three different types as the following Fig 11, Fig 12 and

Fig 13 illustrates.

Thermography methods are not yet used in inline testing of CFRP components. Process-integrated Thermography has to meet a variety of requirements like integrateable, automatable, real time capable, reliable and flexible. The system integration contains the development of strategies and the implementation of the mechanics, electronics, and software for the two main tasks, extern control system and flexible enough for single robot, for TEZ and MFZ work cell. In this paper three different approaches has been proposed for system integration, which demonstrates the advantages and drawbacks (please refer to the figures 9, 10 and11). Finally the scenario 3 has emerged as the optimal method.

The system architecture splits up into 4 main parts: the robot control system (KRC), the Thermography control system including Dim Pack and Flash Generator, the measuring devices including IR camera, halogen lamps and flash lamps and the DLR network system. Each of these four systems contains the relevant electronics, mechanics and software for its special functionality.

Scenario 1 and 2 are very similar. In scenario 1 the Thermography control system is mounted on robot arm (axis no. 3) and measuring devices are mounted on robot hand (axis no. 6). In scenario 2 the both systems are mounted on axis 6 . The advantages of both scenarios are on one hand easy to integrate on robotic cell without changing the system architecture and on the other hand data analysis and measurement performs in real time. But there are several drawbacks for these both scenarios:

a. To include the tools load data in robotic system, the center of gravity of tools is required. Due to the huge and complex construction of measuring system, is difficult to locate it. This can lead to robot inaccuracy

b. To integrate in MFZ more than $50 \mathrm{~m}$ long cable is required for data transmission and power supply

c. This construction does not allow to measure complex components and large measuring volume

d. A heavy weight carrier robot is necessary

Derived from the drawbacks, to achieve high measuring accuracy in large measuring volume on 3D curved components, the measuring system has to be as simple as possible to locate the center of gravity of tool, able to measure and evaluate in real time.

Another approach is the real-time model based quality control by monitoring of process measurable. In order to fulfill the boundary conditions for the tasks, alternative strategies for the implementation of the mechanics, electronics and software into a mechatronic system have been evaluated and implemented. Therefore only the measuring devices are mounted on axis no. 6 and are linked via Ethercat with Thermography control system, which stays on ground. Graphical user interfaces were developed to enable the user to interact with the robot in a comfortable way and to ensure electronic documentation. 


\section{Scenario 1}

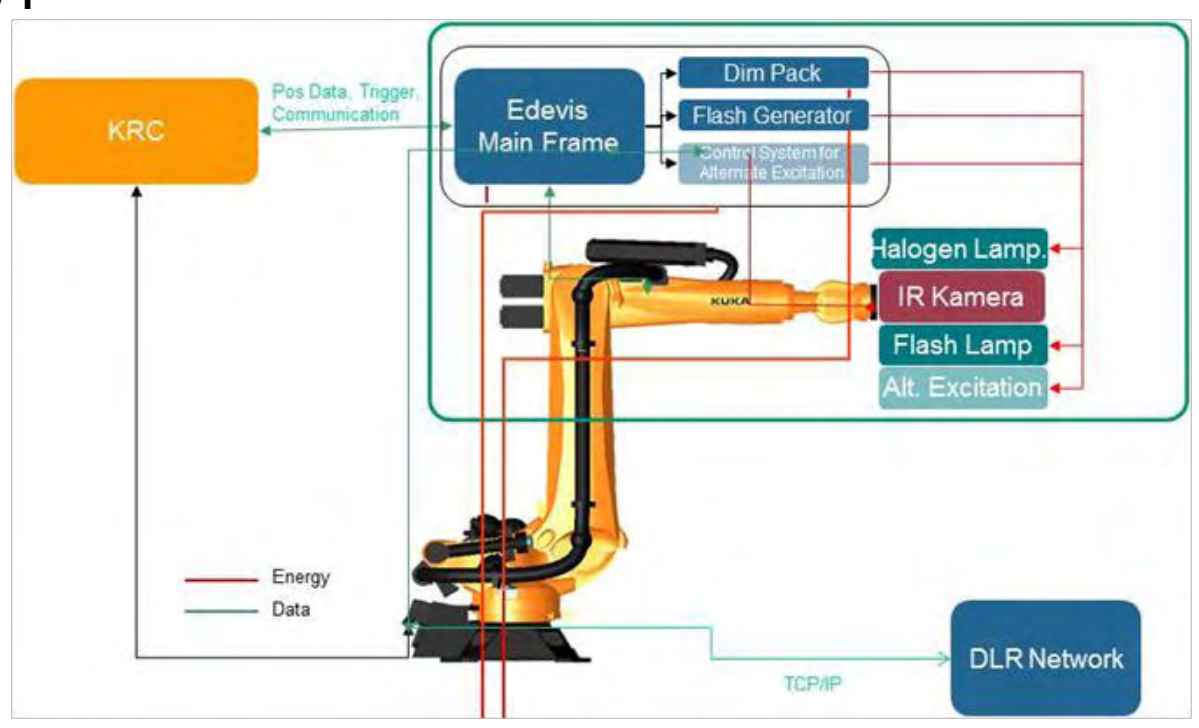

Fig 11 Scenario 1 - Control system mounted on robot arm, excitation and camera on hand

\section{Scenario 2}

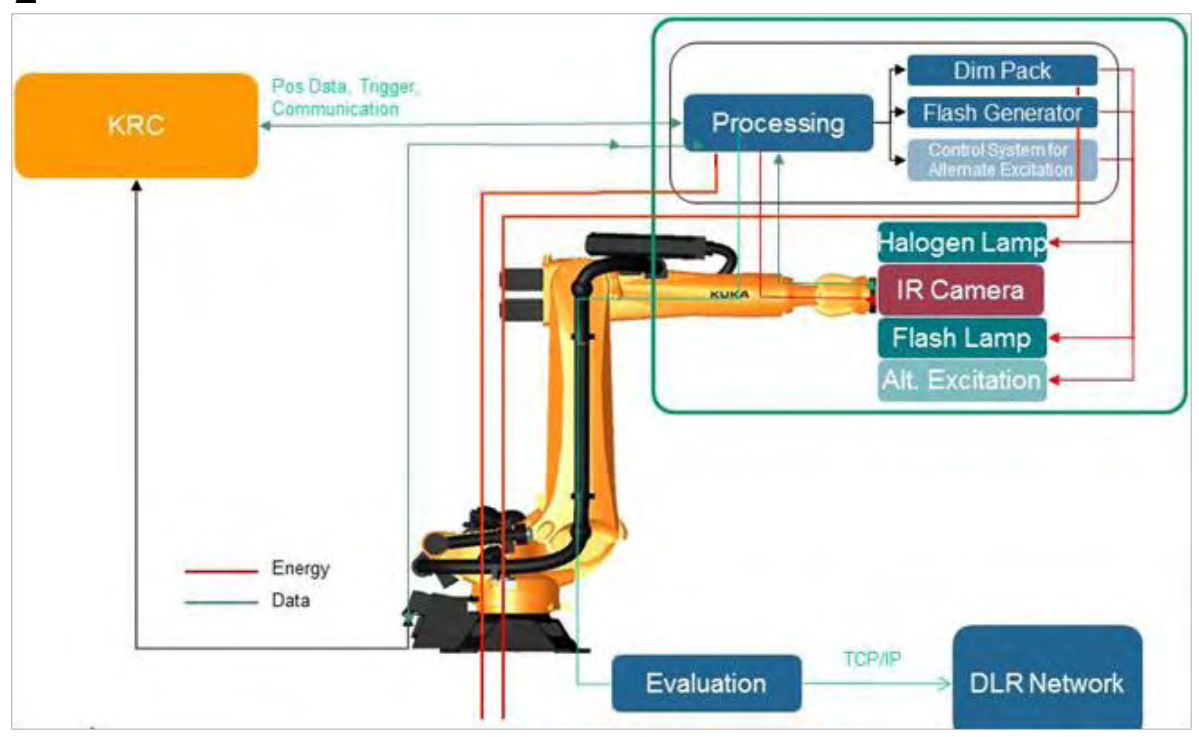

Fig 12 Scenario 2 - Control system, excitation and camera mounted on robot hand 


\section{Scenario 3}

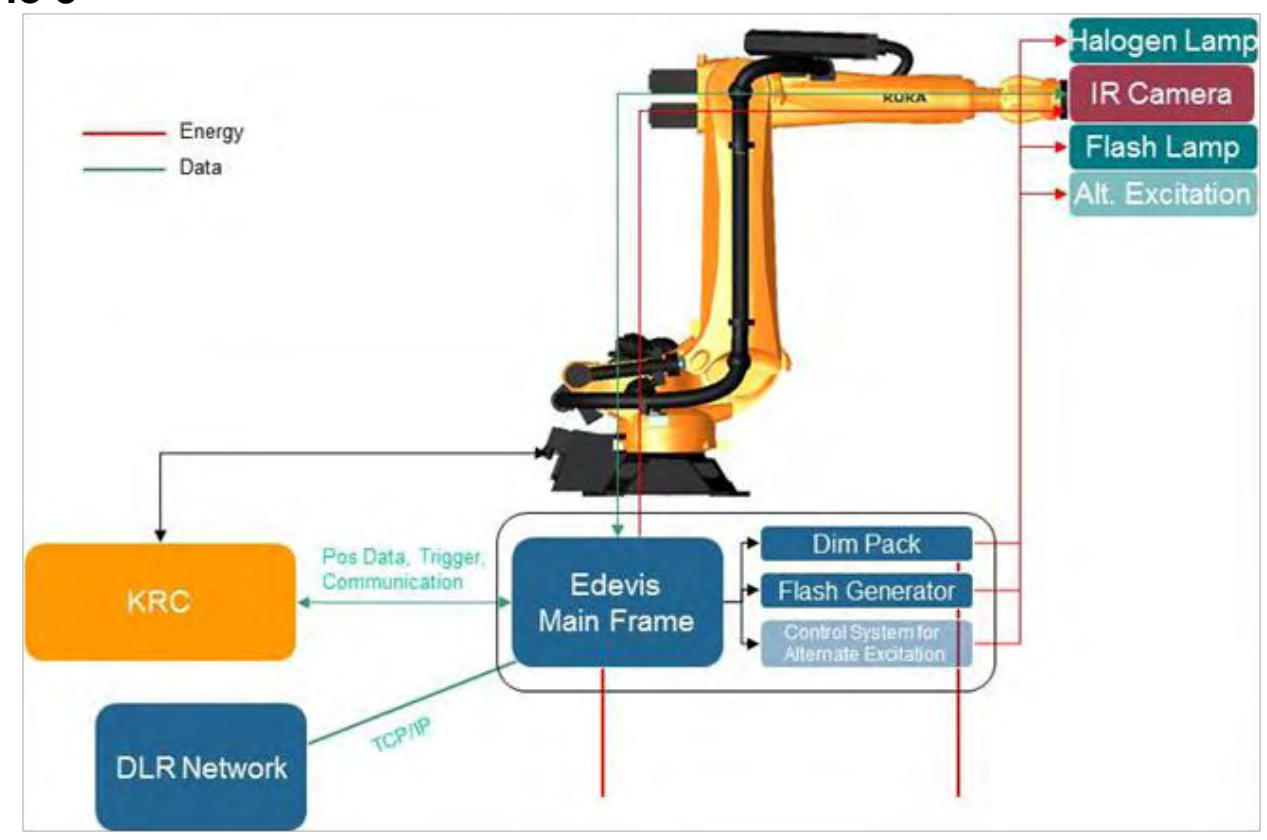

Fig 13 Scenario 3 - excitation and camera mounted on robot hand, control system locally separated

\section{Automation along the process chain}

Fig 14 represents in blue section complete manufacturing process and the closed process below that represents the automated closed monitoring system for cutting, handling and performing manufacturing steps. As an open and closed monitoring tool, it allows to detect and discard all nonconforming components. Only good components go to manufacturing process. Consequently, the process limits can be exhausted, allowing increasing productivity. On the other side, process disturbances affecting the product quality can be detected and located directly. The process can be controlled using its quality characteristics as control variables directly. For this purpose diverse information from CAD Draping simulation and 3D Output are required and used for measurement strategy. For example, to set the measurement parameters and distance in measuring system material properties, components thickness and number of plies are used. After measurement strategy follows the offline programming for path planning relating to robot TCP and as well as to set the camera and halogen position and orientation. All these information are applied to measurement software to perform the measurement step. Getting the information from measurement software an automatic evaluation runs to classify and detect the position of defects. This step verifies the process continuation and keeps all raw data for process understanding.

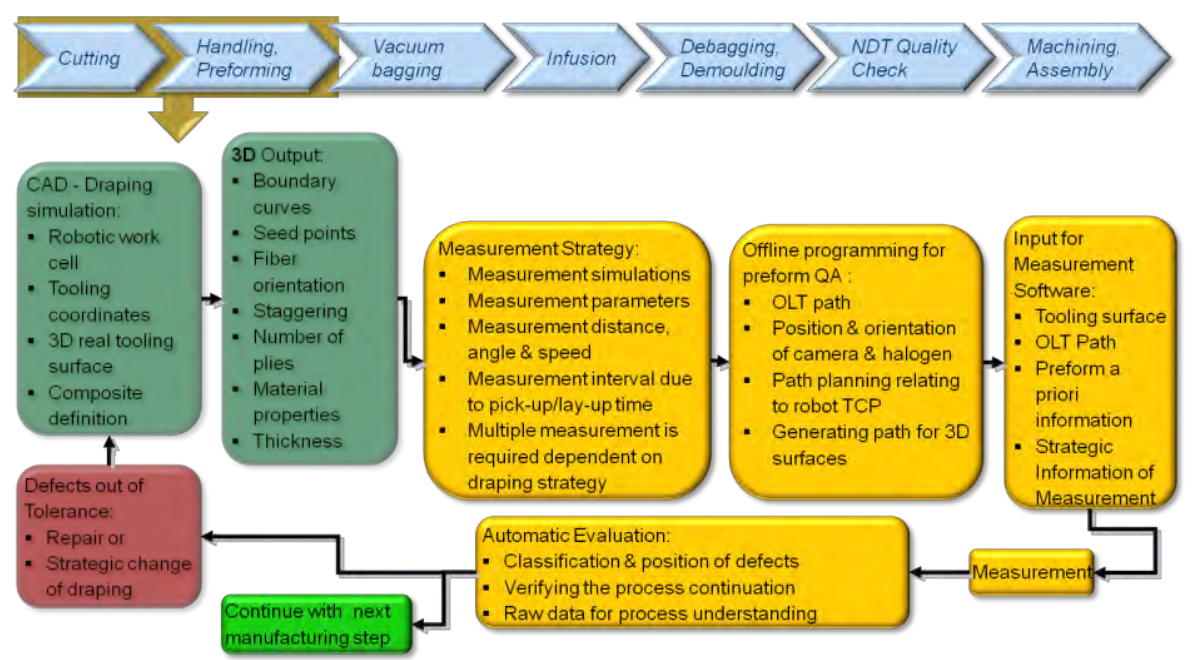

Fig 14 Process monitoring, open and closed loop control with NDT 
In case any kinds of defects are detected, a repair or strategic change is recommended. It is well known, that the integration of different devices and software in this measuring system bring some inaccuracy in the complete measuring system. To have a control on process accuracy every possible failure has been analyzed and presented in Fig 15.

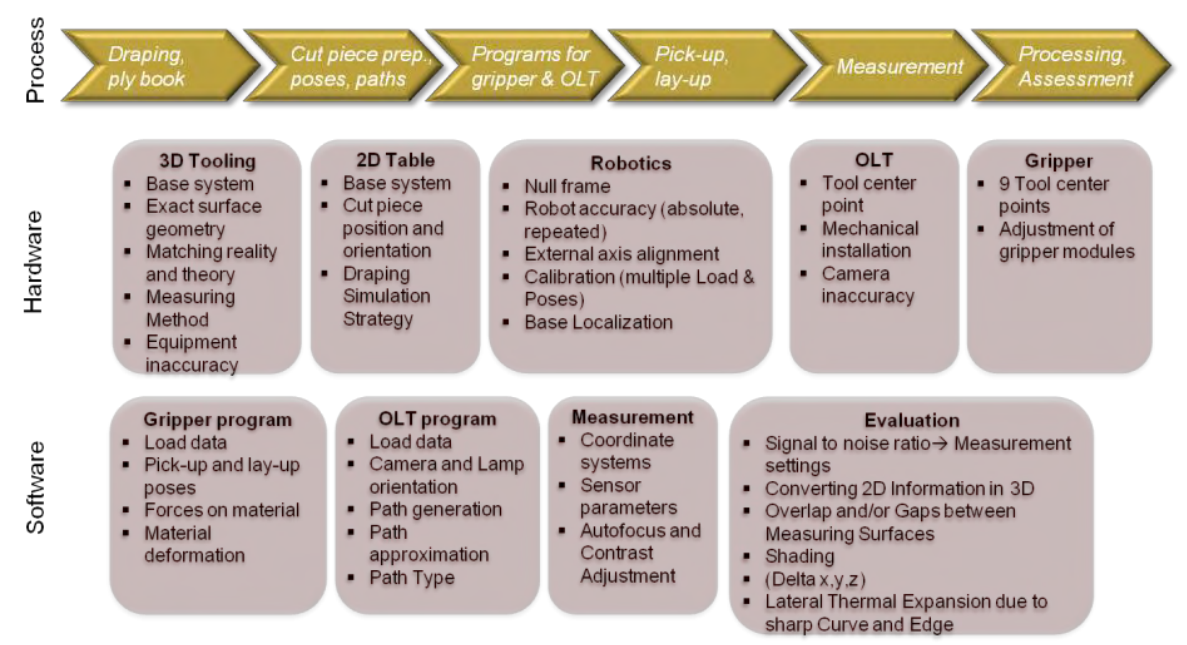

Fig 15 Control on process accuracy

\section{Conclusion}

The case studies have shown, that NDT aided production provides a significant contribution in order to improve product quality, reduce scatter in properties, minimize scrap and improve process economy. In future NDT will be an essential component of a more or less autonomously running production, assuring product and process quality with a minimum of man-machine interaction. As a consequence NDT techniques have to evolve from simple measuring machines to intelligent control systems.

\section{REFERENCES}

[1] Maldague X., O. Moore P. "Infrared and Thermal Testing”. American Society for Nondestructive Testing, Columbus, OH 2001.

[2] Busse G., "Emerging Technologies in Non-Destructive Testing". Proceedings of the 4th International Conference of Emerging Technologies in Non-Destructive Testing (etntd 4), Stuttgart, Germany, 2-4 April 2007 Taylor \& Francis, London, 2008.

[3] Summerscales J., "Non-destructive testing of fibre-reinforced plastics composites 1", Ed. by John Summerscales. Elsevier Applied Science, London, 1987 